\title{
Multiperiodicity of V350 Peg
}

\author{
J. Vidal-Sáinz ${ }^{1}$, P. Wils ${ }^{2}$, P. Lampens ${ }^{3}$, and E. García-Melendo ${ }^{4}$ \\ ${ }^{1}$ Grup d'Estudis Astronomics, Apdo. 9481, 08080 Barcelona, Spain \\ ${ }^{2}$ Vereniging Voor Sterrenkunde, Belgium \\ ${ }^{3}$ Koninklijke Sterrenwacht van België, Ringlaan 3, 1180 Uccle, Belgium \\ ${ }^{4}$ Esteve Duran Observatory Foundation, Montseny 46 - Urb. El Montanya, \\ 08553 Seva, Spain
}

\begin{abstract}
We report on the variability of V350 Peg, a $\delta$ Scuti star newly discovered by Hipparcos. Based on observations acquired during 35 nights at two private observatories in Spain, we could show that this star pulsates in a close doublet of frequencies $(\Delta \mathrm{f} / \mathrm{f}=3 \%)$. The period as listed in the Hipparcos Catalogue is not confirmed.
\end{abstract}

V350 Peg (= HIP 115563) is a small-amplitude variable star assigned to the $\delta$ Scuti class of pulsating stars with a period of 0.2012 days and a total amplitude of 0.05 mag (ESA 1997). During 35 nights between July 1997 and December 2001 some 7500 CCD differential observations in the $V$ band were acquired at the Monegrillo and Esteve Duran observatories in Northern Spain. HD 220538 (= HIP 115545) served as a comparison star.

A Fourier analysis of the new data revealed two significant frequencies separated by $0.17 \mathrm{c} / \mathrm{d}$ and with very similar amplitudes: one at $5.840 \mathrm{c} / \mathrm{d}(0.1712 \mathrm{~d}$; semi-amp. $27 \mathrm{mmag})$ and one at $5.668 \mathrm{c} / \mathrm{d}(0.1764 \mathrm{~d}$; semi-amp. $24 \mathrm{mmag})$. The result is an obvious beat phenomenon with a period of 5.81 days. But a fit with only two frequencies explains only half of the total variation (Fig. 1). The ratio of the most dominant frequencies $\left(f_{2} / f_{1}=0.97\right)$ indicates non-radial pulsation for at least one of the modes. More frequencies are obviously excited. Though we have been searching for them, we cannot be confident about their correct identification due to various kinds of aliasing effects. The detailed anal- 
ysis will soon be available (Vidal-Sáinz et al. 2002).

The multiperiodicity combined with the short beat period and the fact that several non-radial modes are possibly excited, make this star a worthwhile target for a follow-up study in the context of a multisite campaign.

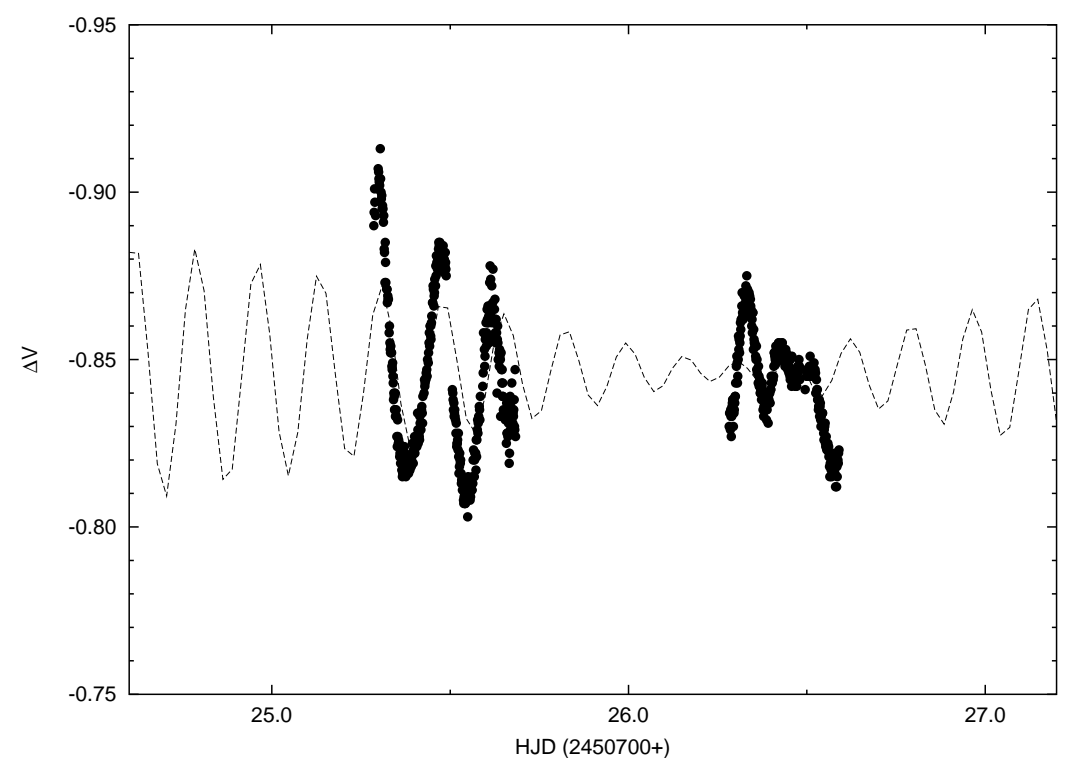

Figure 1: CCD data of V350 Peg on consecutive nights, and two-frequency fit

\section{References}

ESA 1997, The Hipparcos and Tycho Catalogues, ESA SP-1200

Vidal-Sáinz J., Wils P., Lampens P., García-Melendo E., A\&A, accepted for publication 